\title{
Studies of Dilepton Production in Coarse-Grained Transport Dynamics
}

\author{
Stephan Endres* ${ }^{\dagger}$ \\ Universität Frankfurt, Max-von-Laue-Straße 1, 60438 Frankfurt, GERMANY \\ Frankfurt Institute for Advanced Studies, Ruth-Moufang-Straße 1, 60438 Frankfurt, GERMANY \\ E-mail: endresath.physik.uni-frankfurt.de
}

\section{Hendrik van Hees}

Frankfurt Institute for Advanced Studies, Ruth-Moufang-Straße 1, 60438 Frankfurt, GERMANY

E-mail: hees@fias.uni-frankfurt.de

\section{Marcus Bleicher}

Universität Frankfurt, Max-von-Laue-Straße 1, 60438 Frankfurt, GERMANY

Frankfurt Institute for Advanced Studies, Ruth-Moufang-Straße 1, 60438 Frankfurt, GERMANY

E-mail: bleicher@th.physik.uni-frankfurt.de

\begin{abstract}
As microscopic transport models usually have difficulties to deal with in-medium effects in heavy-ion collisions, we present an alternative approach that uses coarse-grained output from transport calculations with the UrQMD model to determine thermal dilepton emission rates. A four-dimensional space-time grid is set up to extract local baryon and energy densities, respectively temperature and baryon chemical potential. The lepton pair emission is then calculated for each cell of the grid using thermal equilibrium rates. In the current investigation we inlcude the medium-modified $\rho$ spectral function by Eletsky et al., as well as contributions from the QGP and four-pion interactions for high collision energies. First dielectron invariant mass spectra for $\mathrm{Au}+\mathrm{Au}$ collisions at $1.25 \mathrm{AGeV}$ and for dimuons from In+In at $158 \mathrm{AGeV}$ are shown. At 1.25 $\mathrm{AGeV}$ a clear enhancement of the total dilepton yield as compared to a pure transport result is observed. In the latter case, we compare our outcome with the NA60 dimuon excess data. Here a good agreement is achieved, but the yield in the low-mass tail is underestimated. In general the results show that the coarse-graining approach gives reasonable results and can cover a broad collision-energy range.
\end{abstract}

8th International Workshop on Critical Point and Onset of Deconfinement,

March 11 to 15, 2013

Napa, California, USA

\footnotetext{
* Speaker.

${ }^{\dagger}$ This work has been supported by the Helmholtz Research School for Quark-Matter Studies (H-QM), the Helmholtz International Center for FAIR (HIC for FAIR) and BMBF.
} 


\section{Introduction}

Lepton pairs at low mass are considered good direct probes for the hadronic properties of hot and dense nuclear matter. They may deliver information considering chiral symmetry breaking and the in-medium properties of vector mesons [1]. In experiments with focus on low-mass dileptons (as HADES at GSI, NA60 at CERN SPS or PHENIX and STAR at RHIC) an enhancement in heavy systems was observed in the low mass regime, when comparing data to hadronic cocktail calculations [2, 3]. Although several ideas $[4,5]$ have been offered to explain these results, they are not yet fully understood.

A main challenge for the theoretical grasp of dilepton production is the broad variety of sources. It requires models that can describe all the different channels which contribute to the spectra. One frequent approach is the use of microscopic transport calculations, as e.g. realized by the UrQMD model [6, 7]. However, transport models usually have problems to handle in-medium effects, as a non-equilibrium treatment of off-shell effects and the realization of multi-particle scatterings is difficult. In these proceedings we present a different approach that uses coarse-grained output from UrQMD calculations to extract local thermodynamic properties and calculate thermal dilepton rates. Similar studies were carried out before, e.g. by employing hydrodynamics or a combined transport-hydro hybrid approach [8,9]. Nevertheless, the coarse-graining has the advantage that it can cover all collision energies and the whole space-time evolution in a unified description.

\section{Coarse-graining approach}

For our calculations, we take an ensemble of UrQMD events and span a grid of small spacetime cells, for which a spatial length of $1 \mathrm{fm}$ and a time resolution of 0.5 to $1 \mathrm{fm}$ is chosen. By putting the UrQMD output on the grid, we get the baryon number and total energy for each cell. To extract the local rest frame baryon and energy densities we calculate the baryon four-flow $j_{B}^{\mu}$ and perform a Lorentz boost so that $\overrightarrow{j_{B}}=0$ (Eckart frame). Temperature $T$ as well as baryon and quark chemical potential $\mu_{B / q}$ are then determined by the use of a tabulated equation of state (EoS). At low collision energies a pure hadron gas approach is sufficient [10], while a chiral EoS including a phase transition and chiral symmetry restoration is needed for our NA60 calculations [11].

Taking vector meson dominance into account, the thermal dilepton rate can be related to the spectral properties of the vector meson. The lepton-pair emission is then calculated for each cell of the grid using thermal equilibrium rates per four-volume and four-momentum [12],

$$
\frac{\mathrm{d}^{8} N_{V \rightarrow l l}}{\mathrm{~d}^{4} x \mathrm{~d}^{4} q}=-\frac{\alpha^{2} m_{V}^{4}}{\pi^{3} g_{V}^{2}} \frac{L\left(M^{2}\right)}{M^{2}} f_{B}\left(q_{0} ; T\right) \operatorname{Im} D_{V}^{(\mathrm{ret})}\left(M, q ; T, \mu_{B}\right) .
$$

In our current analysis only the contribution from the $\rho$ meson is considered, as it is expected to show the largest medium modifications. For the in-medium self energy we apply the description by Eletsky [13] which uses empirical scattering amplitudes from resonance dominance at low energies and Regge-type behaviour at high energies.

When considering SPS energies, it is necessary to additionally include contributions that stem from the QGP phase and from 4-pion processes. The $4 \pi$ lepton-pair production can be determined from the electromagnetic spectral function extracted in $e^{+} e$ annihilation [14], while the QGP contribution is evaluted according to the $q \bar{q}$ annihilation rate [15]. 

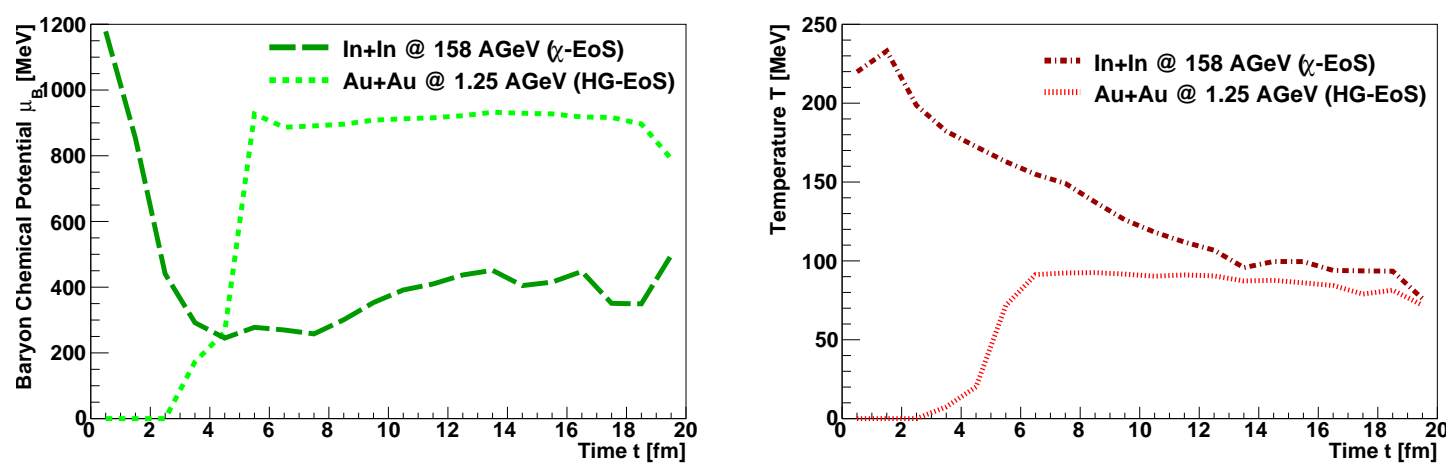

Figure 1: Time evolution of $\mu_{B}$ (left) and $T$ (right) for a cell at the center of the collision. The results for central $\mathrm{Au}+\mathrm{Au}$ collisions with $E_{\mathrm{lab}}=1.25 \mathrm{AGeV}$ and for $\mathrm{In}+\mathrm{In}$ at $E_{\mathrm{lab}}=158 \mathrm{AGeV}(b<9 \mathrm{fm})$ are shown. A hadron gas EoS $(\mathrm{Au}+\mathrm{Au})$ respectively a chiral $\operatorname{EoS}(\mathrm{In}+\mathrm{In})$ was used to determine $\mathrm{T}$ and $\mu_{B}$.

\section{Results}

Fig. 1 shows the time evolution of chemical potential and temperature for a cell at the center of the collision. While we get a mamixum temperature of about $100 \mathrm{MeV}$ at an energy of $1.25 \mathrm{AGeV}$, it is significantly higher for $E_{\mathrm{lab}}=158 \mathrm{AGeV}$ and reaches up to $240 \mathrm{MeV}$ in this case. Considering the low energy collisions, the baryon chemical potential remains on a high level ( $\sim 900 \mathrm{MeV})$ after the inititial increase, while it drops rapidly after the start of the collision and then remains at relatively low values for the NA60 calculation. In general we get reasonable values for both quantities which are in line with what is expected. For $158 \mathrm{AGeV}$ the results are also in good agreement with previous studies [9].

Looking at the dilepton invariant mass spectra (Fig. 2), we see that for the case of $\mathrm{Au}+\mathrm{Au}$ at $1.25 \mathrm{GeV}$ the peak structure of the $\rho$ vanishes and the whole spectral function gets a Dalitz type shape. With the in-medium $\rho$, the total yield is a factor of 2-3 higher in the mass region from 200 to $600 \mathrm{MeV}$ than what we obtain from a vacuum calculation. Note that in the latter we additionally included the UrQMD contribution from the Dalitz decay of the $\Delta_{1232}$, which is already contained in the in-medium $\rho$ as a subthreshold contribution.

For NA60 the Eletsky spectral function gives a good overall agreement and describes the excess spectrum very well at the pole mass of the $\rho$, but it underestimates the low-mass tail. This has been observed in other investigations as well and seems to be a general problem of the Eletsky spectral function [3]. Above $1 \mathrm{GeV}$ the contributions from the QGP and 4-pion interactions become dominant. Here again the calculation gives a significantly lower result than measured in experiment, especially the QGP contribution seems to be rather low. However, not all sources that might contribute are yet included in the calculation, e.g. thermal $\omega$ and $\phi$ contributions are missing.

\section{Summary and Conclusion}

We presented first dilepton spectra from coarse-grained transport dynamics for HADES and NA60 energies. The results show that this unified approach of dilepton production can cover a broad collision-energy range. The combination of a realistic transport model with in-medium 

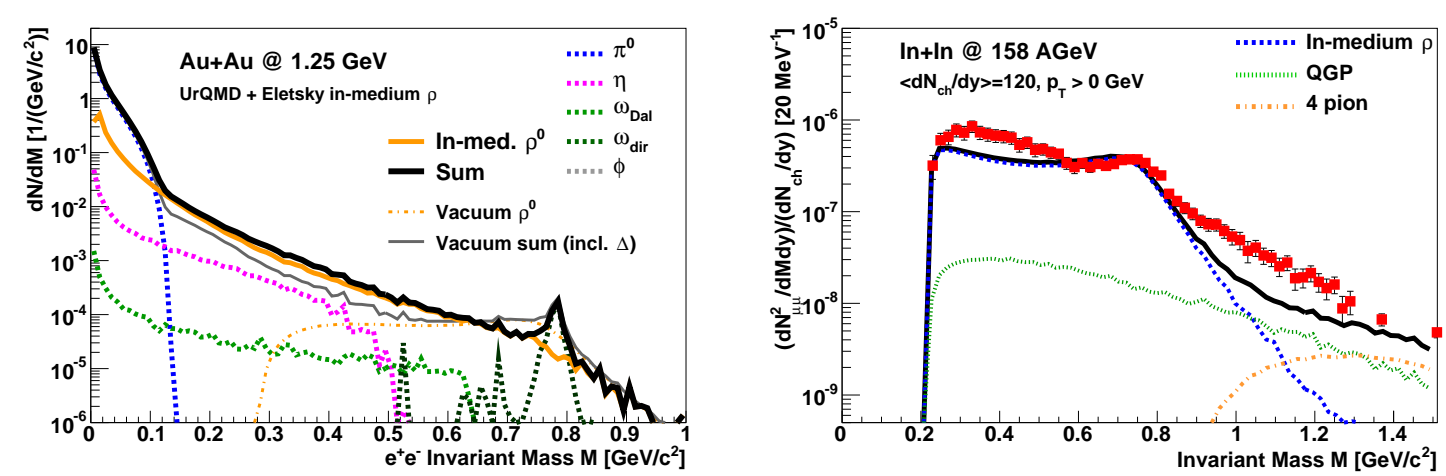

Figure 2: (Left) Dielectron invariant mass spectrum for central $\mathrm{Au}+\mathrm{Au}$ collisions at $E_{\mathrm{lab}}=1.25 \mathrm{AGeV}$. The thermal contribution of the in-medium modified $\rho$ from coarse-graining is plottet together with the pure transport results for the other direct or Dalitz decays. For comparison we also show the result for a vacuum $\rho$ spectral function. (Right) Dimuon excess spectrum from coarse-grained dynamics for $\operatorname{In}+\operatorname{In}$ at $E_{\mathrm{lab}}=158$ $\mathrm{AGeV}$ with $b<9 \mathrm{fm}$, corresponding to $\left\langle\mathrm{d} N_{c h} / \mathrm{d} y\right\rangle=120$. The results are compared to data from NA60 [3].

spectral functions enables a better description of medium modifications than with conventional microscopic calculations. However, we see that the NA60 data are not yet fully described with the current setting, especially in the low-mass tail. Further investigations on this with different spectral functions and equations of state are in preparation. Besides, it will be instructing to compare our outcome with the pending results on $\mathrm{Au}+\mathrm{Au}$ reactions at $1.25 \mathrm{AGeV}$ from the HADES Collaboration. The coarse-graining dilepton spectra indicate that we should expect to see a significant excess in the intermediate mass range due to medium modifications of the $\rho$ meson.

\section{References}

[1] C. Gale and J. I. Kapusta, Phys. Rev. C 35 (1987) 2107.

[2] G. Agakishiev et al. (HADES Collab.), Phys. Rev. C 84 (2011) 014902, [arXiv:1103.0876].

[3] R. Arnaldi et al. (NA60 Collab.), Eur. Phys. J. C 61 (2009) 711, [arXiv:0812.3053].

[4] G. E. Brown and M. Rho, Phys. Rev. Lett. 66 (1991) 2720.

[5] R. Rapp, G. Chanfray and J. Wambach, Nucl. Phys. A 617 (1997) 472.

[6] S. A. Bass et al., Prog. Part. Nucl. Phys. 41 (1998) 255, [nucl-th/9803035].

[7] K. Schmidt et al., Phys. Rev. C 79 (2009) 064908, [arXiv:0811.4073].

[8] E. Santini et al., Phys. Rev. C 84 (2011) 014901, [arXiv:1102.4574].

[9] P. Huovinen et al., Phys. Rev. C 66 (2002) 014903, [nucl-th/0203023].

[10] D. Zschiesche et al., Phys. Lett. B 547 (2002) 7, [nucl-th/0209022].

[11] J. Steinheimer, S. Schramm and H. Stocker, J. Phys. G 38 (2011) 035001, [arXiv:1009.5239].

[12] R. Rapp and J. Wambach, Adv. Nucl. Phys. 25 (2000) 1, [hep-ph/9909229].

[13] V. L. Eletsky et al., Phys. Rev. C 64 (2001) 035202, [nucl-th/0104029].

[14] Z. Huang, Phys. Lett. B 361 (1995) 131, [hep-ph/9506399].

[15] J. Cleymans, J. Fingberg and K. Redlich, Phys. Rev. D 35 (1987) 2153. 